\title{
The pedicled omentoplasty and split skin graft (POSSG) for reconstruction of large chest wall defects. A validity study of 34 patients
}

\author{
Caroline M. E. Contant*, Albert N. van Geel*, Bronno van der Holt $\dagger$ and \\ Theo Wiggers* \\ *Department of Surgical Oncology and $\dagger$ Department of Statistics, Academic Hospital Rotterdam-Dr. Daniel \\ den Hoed Cancer Center; The Netherlands
}

\begin{abstract}
The aim of this study was to evaluate retrospectively the results of pedicled omentoplasty and split skin graft (POSSG) in reconstructing (full thickness) chest wall defects, and to define its role as a palliative procedure for local symptom control. Thirty-four patients with recurrent breast cancer $(n=25)$, radiation-induced necrosis $(n=5)$ or sarcoma $(n=4)$ of the chest wall were selected for the study. All patients underwent curative or palliative chest wall resection with reconstruction by pedicled omentoplasty and split skin graft (POSSG), between 1986 and 1994. Reconstructive outcome, complications, local tumour and symptom control following surgery was measured. The most common complication was shown to be partial necrosis of the omental flap (35\%), followed by respiratory problems $(26 \%)$, facial hernia $(26 \%)$ and thoracic wound problems $(15 \%)$, which were mostly treated in a conservative way $(68 \%)$. The 3 -year local tumour-free interval after POSSG in patients curatively treated for breast cancer is $16 \%$. Seventy per cent of the patients who underwent palliative resection had longstanding relief of local pain, bleeding or foetor due to local tumour growth. It can be concluded that large (full thickness) chest wall defects after resection of local recurrence, primary malignancy or osteoradionecrosis of the chest wall can successfully be reconstructed by POSSG. Chest wall resection in patients treated with palliative intention is effective in local symptom control.
\end{abstract}

Key words: omentoplasty; chest wall reconstruction.

\section{Introduction}

Sarcomas of the chest wall, local recurrent breast cancer and radiation-induced necrosis are the main indications for chest wall resection. Depending on local tumour growth or radionecrotic lesions large areas of soft tissue. including the skin, may have to be resected as well as parts of the skeletal chest wall. In both circumstances reconstructive procedures have to be performed as primary closure is impossible.

Soft tissue coverage of the chest wall can be achieved by the use of myocutaneous flaps, such as the rectus abdominis, the latissimus dorsi or the pectoralis major flap with or without microsurgical anastomosis. For this group of patients this technique has several disadvantages. Previous operation could have damaged the vascular bundle of the flap, which is also at risk in patients who are treated with radiotherapy after chest wall resection. Another disadvantage is the duration of this surgical procedure.

Kiricuta was the first who proposed omental transposition with split skin graft to repair these soft tissue defects.' The pedicled omentoplasty has the following advantages. Due to its angiogenic and anti-inflammatory properties the omentum can be successfully transposed to areas with chronic infection .

Correspondence to: A. N. van Geel, PhD, Department of Surgical Oncology, Academic Hospital Rotterdam-Dr. Daniel den Hoed Cancer Center, Groene Hilledijk 30I, 3075 EA Rotterdam, The Netherlands. and lack of blood supply as a result of radiation damage and has proven to be an excellent base for skin grafting. The pliability of the omentum gives no limitations to the acceptor zone.

For skeletal reconstruction the use of absorbable materials like polyglactine (Vicryl®) or homologous dura mater (Lyodura ${ }^{\circledR}$ ) is preferable because non-absorbable materials like polypropylene (Marlex®) increase the risk of chronic infection, especially in restoration of radiation ulcers. ${ }^{2-4}$

For the above-mentioned reasons we decided to reconstruct large chest wall defects by a uniform approach using pedicled omentoplasty and split skin graft (POSSG) in combination with absorbable materials to achieve stability of the skeletal chest wall defects.

This study was performed to evaluate our results, focusing on post-operative complications of POSSG and on local tumour control for patients who are treated with curative intention. Furthermore, an attempt has been made to define the role of POSSG as a palliative procedure for local symptom control.

\section{Patients and methods}

A retrospective review was performed of 34 patients with partial or full thickness chest wall resection for breast cancer or sarcoma of the chest wall, between December 1986 and 


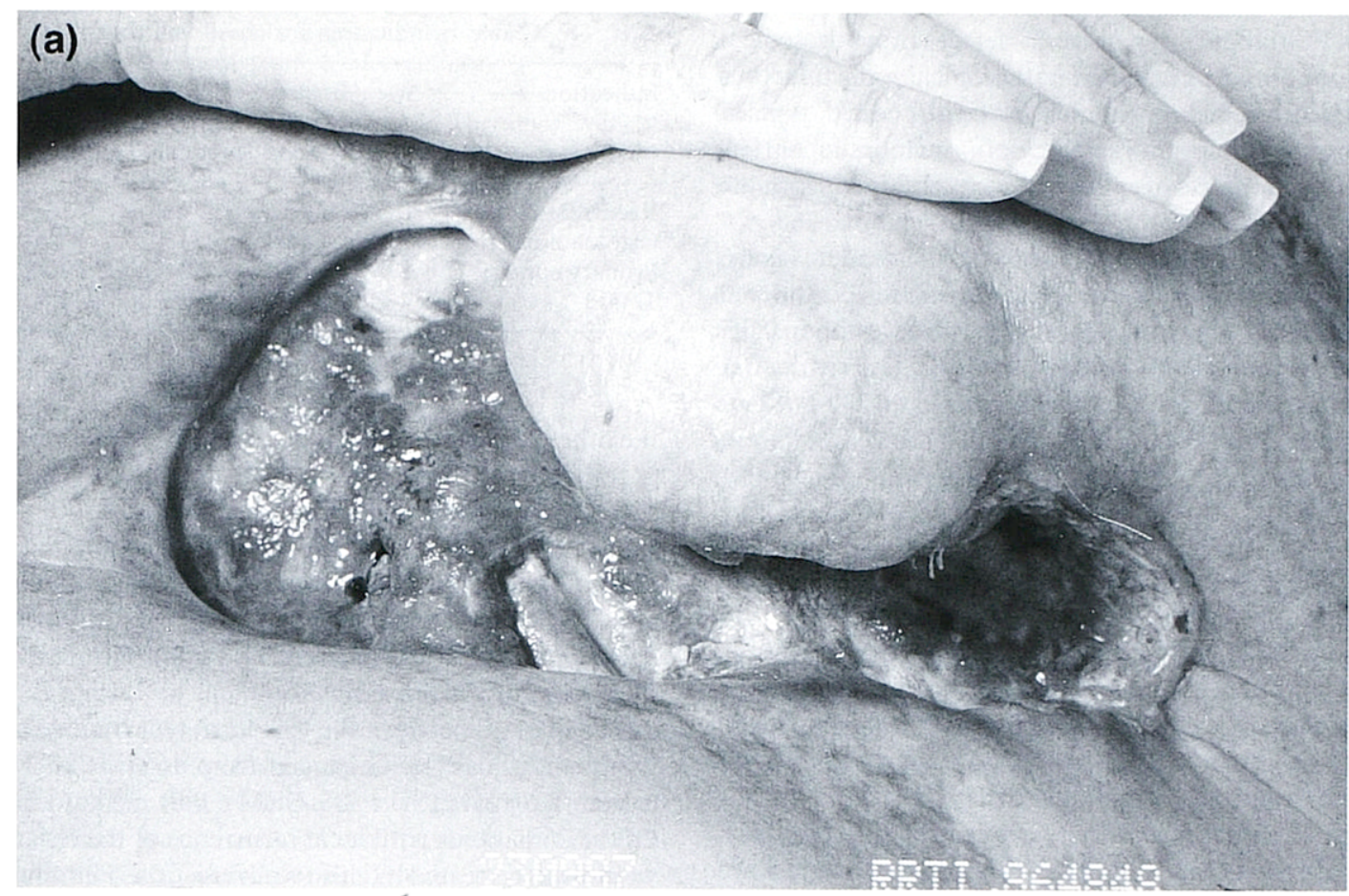

(b)

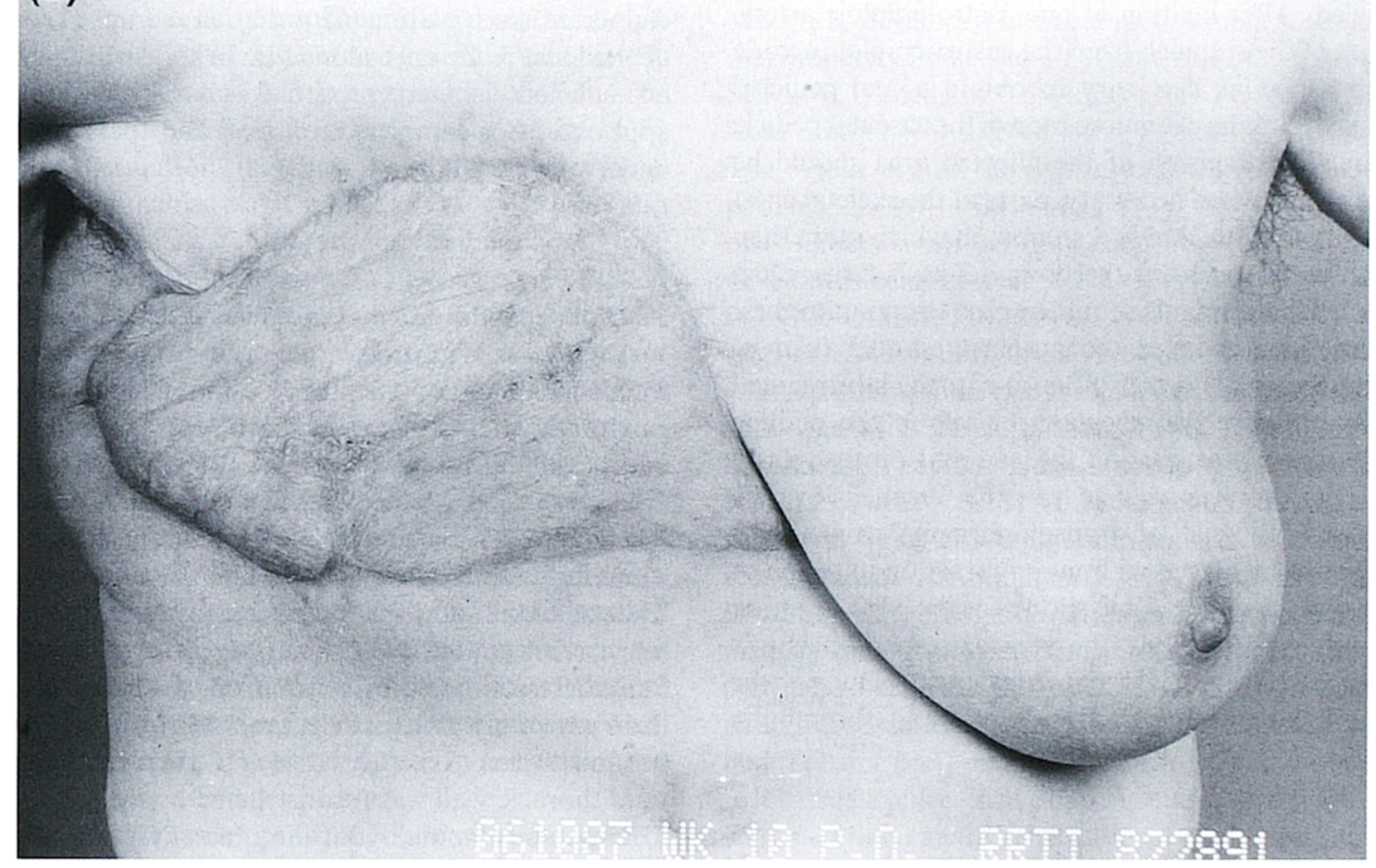

Fig. 1. (a) A large ulcerating breast tumour, initially treated with chemotherapy and radiation therapy, which showed no tumour regression. Partial chest wall resection was the final option. (b) Reconstruction with POSSG; result 2 months after operation.

December 1994 in the Academic Hospital Rotterdam-Dr. Daniel den Hoed Cancer Center. Chest wall resection was carried out with curative intention, in case of osteoradionecrosis, as a primary treatment, or if radiotherapy, hormonal or chemo-therapy had failed to control local recurrence and no distant metastases were found (Fig. 1). Palliative resection was indicated in patients with distant metastases or mastitis carcinomatosis, because of the severe local pain, bleeding or odorous ulceration due to local tumour growth. All chest wall resections are performed only when macroscopic adequate margins could be achieved.

The clinical records were studied for data regarding previous treatment, indication for chest wall resection, surgical procedure, complications, adjuvant treatment and 
follow-up. Histological type, size of defect and pathological margins were obtained from the pathological report. Details of the surgical procedure (i.e. left or right pedicle, type of laparotomy, route to thoracic region, soft tissue or full thickness resection, use of implants) were obtained from the operating surgeon's report.

Fisher's exact test was used for the statistical calculations. Differences between observed groups were considered statistically significant with two-sided $P$-values less than 0.05 . Kaplan-Meier curves of local control and survival after omental transposition were constructed. ${ }^{5}$

\section{Sirgical technique}

Following laparotomy the abdominal cavity, liver and the omentum are inspected for metastatic deposits. When no extensive intra-abdominal metastatic spread of liver or omentum is found, the omentum is detached from the transverse and meso-colon. A decision has to be made whether the right or left gastro-epiploic artery should be divided. The right gastro-epiploic artery is larger and has more epiploic branches and therefore is generally used as a nutrient vessel. When both gastro-epiploic arteries are available. the side of acceptor place on the chest wall corresponds with the artery on which the omentoplasty has to be pedicled. After ligation of one gastro-epiploic artery, the branches of the stomach from the gastro-epiploic arcade are divided as far as necessary to obtain a real pedicled omentoplasty. Kinking or compression of the vascular pedicle must be avoided. Excision of the affected area should be radical. including, when necessary. parts of the skeletal chest wall. In cases of full thickness resection, in which more than one rib is resected or when the bony defect is larger than $50 \mathrm{~cm}^{2}$, absorbable implants should be used to maintain chest wall stability. The pedicled omentoplasty should then be transposed from the abdomen. Usually. an artificial incisional hernia with sufficient diameter can be left in the midline laparotomy wound for passing the pedicled omentoplasty through. The thoracic region can be reached by the subcutaneous, subfacial or transdiaphragmal route. The omentum has to be placed without any traction in the chest wall defect and fixed with some sutures at the edges. During the same surgical procedure the pedicled omentoplasty is covered by a 1:3 meshed split-skin graft." During the operation all patients received antibiotic and thrombotic prophylaxis.

\section{Results}

Thirty-four patients, 31 women and 3 men, underwent transposition of the greater omentum to reconstruct chest wall defects. The age ranged from 28 to 91 years (mean: 56.5 years $(S D=13.7, S E M=2.3$ ) median: 54.4 years).

The indications for chest wall surgery were recurrent cancer in 26 (breast cancer: 24; sarcoma: 2), osteoradionecrosis without clinical evidence of recurrence in five and primary surgery in three patients (breast cancer: 1; sarcoma: 2). Twenty-four patients were treated with curative intention and
Table 1. Indication for chest wall resection.

\begin{tabular}{lrrrrr}
\hline Indication & $N$ & $\begin{array}{c}\text { Breast } \\
\text { cancer }\end{array}$ & $\begin{array}{c}\text { Soft } \\
\text { tissue } \\
\text { satrcomal }\end{array}$ & \multicolumn{2}{c}{ Intention } \\
\cline { 5 - 6 } & & & Curative Palliative \\
\hline Recurrent cancer & 26 & 24 & 2 & 16 & 10 \\
Osteoradionccrosis & 5 & 5 & - & 5 & - \\
Primary surgery & 3 & 1 & 2 & 3 & - \\
Total & 34 & 30 & 4 & 24 & 10 \\
\hline
\end{tabular}

the other 10 patients underwent palliative resection (Table 1).

Initial treatment consisted of surgery (seven patients). surgery in combination with radiotherapy (20), exclusive radiotherapy (2) and radiotherapy in combination with chemotherapy (2). The interval between the initial treatment and POSSG ranged from 0.3 to 22.4 years (mean: 6.7 years $(\mathrm{SD}=6.4, \mathrm{SEM}=1.1$ ) median: 5.0 years). Three patients had POSSG as primary treatment.

The interval between the first local recurrence after initial treatment and POSSG ranged from 10 days to 11.9 years (mean: 2.6 years $(\mathrm{SD}=3.5 . \mathrm{SEM}=0.7$ ) median: 0.9 years). Of the 26 patients with local recurrence of the chest wall, six were directly treated by chest wall resection. The remaining 20 patients were treated with radiation therapy in combination with chemo- or hormonal-therapy (10), cytotoxic or endocrine manipulation (6), radiation therapy (1) or excision of the local recurrence alone (3). In spite of these therapies no sufficient local regression was achieved or recurrence appeared after complete remission and these patients then underwent POSSG.

\section{Treament}

In 19 patients the omentoplasty was pedicled on the right, in 11 on the left gastro-epiploic artery. Two patients had transposition of the omentum without ligation of one of the gastro-epiploic arteries. In the surgeon's report of two patients no information was given whether the right or left artery was used as a pedicle. The omentoplasty reached the thoracic region by subcutaneous route in 20 . by subfacial route in five and by transdiaphragmal route in one patient. The size of the soft tissue defect ranged from 16.5 to $789 \mathrm{~cm}^{2}$ (mean: $208 \mathrm{~cm}^{2}(S D=173, S E M=30)$ median: $153 \mathrm{~cm}^{2}$ ). Skeletal resection of ribs, sternum or clavicula was performed in 16 patients, leaving a defect ranging from 10.5 to $234 \mathrm{~cm}^{2}$ (mean: $90 \mathrm{~cm}^{2}(\mathrm{SD}=62, \mathrm{SEM}=16)$ median: $\left.98 \mathrm{~cm}^{2}\right)$. The rigid thoracic wall was reconstructed by the use of implants in 13 patients; homologous dura mater (Lyodura $($ ) in nine, polyglycolic acid (Dexon®) in two and propylene (Marlex®) in two patients. Three patients did not have skeletal reconstruction of the chest wall defect, whether the defect was small (2) or only the clavicula was resected. No intraoperative complications occurred during preparation of the omentoplasty and reconstruction of the chest wall defect.

After chest wall resection additional therapy was given in nine patients: radiotherapy in five and chemo- or hormonal therapy in four. Indications for adjuvant radiotherapy were resection of sarcoma in two and irradical resection in three patients. Two patients had started chemotherapy just before chest wall resection and continued it afterwards. 
Table 2. Post-operative complications following POSSG and subsequent surgical re-intervention

\begin{tabular}{|c|c|c|c|c|}
\hline \multirow[t]{2}{*}{ Complication } & \multirow[t]{2}{*}{$N$} & \multirow[t]{2}{*}{$(\%)$} & \multicolumn{2}{|c|}{ Therapy } \\
\hline & & & Conservalive & Surgical \\
\hline Partial necrosis & 12 & (35) & 12 & \\
\hline Respiratory & 9 & (26) & 9 & - \\
\hline Hernia & 9 & (26) & 3 & 6 \\
\hline Thoracic wound & 5 & (15) & 2 & 3 \\
\hline Abdominal wound & 3 & (9) & 1 & 2 \\
\hline Total & 37 & & 27 & 11 \\
\hline
\end{tabular}

\section{Post-operative complications}

Ten patients $(29 \% 1 \%)$ had a completely uneventful postoperative course. In 11 patients one to three surgical reinterventions after the chest wall reconstruction were necessary as a result of the thoracic wound (3). hernia (6) or abdominal wound (2) (Table 2).

Before 1991 division of the omental pedicle 6 weeks after POSSG was routine and at the same surgical procedure the incisional hernia was closed." Four patients underwent correction of the fascial defect for this reason. In one of these pattients a metastatic deposit in the omental pedicle was found, which was proved-by histological examination. Since 1991 four patients have had complaints about their incisional hernia. Two patients were conservatively treated. one patient had surgical correction and another patient is scheduled for operation to correct the hernia. There was no difference in incidence of herniation whether the omentoplasty was tunnelled subcutaneously or sublacially to the thoracic region. Another patient had a transdiaphragmal herniation of the stomach. which was diagnosed by roentgenological examination. The artificial hernia of the diaphragma was reconstructed by an interior gastropexy I week after chest wall reconstruction.

Twelve patients $(35 \%)$ developed partial necrosis of the omental flap: less than $10 \%$ in seven and $10-33 \%$ in five. In each group four patients needed necrotectomy: in the group with less than 10\% necrosis necrotectomy was necessary only once, in the group with 10-33\% necrosis necrotectomy was performed in two procedures. Secondary wound healing after removal of the necrosis was seen in 2910134 days (mean: 66 days ( $S D=36, S E M=10$ ) median: 53 days) alfer POSSG. There was no relation between the size of delect or artery used as the nutrient vessel, and the development of necrosis of the omental flap.

Complications in relation to the thoracic wound occurred in five patients $(15 \%)$. A bscess formation behind the omental flap was caused by infection of the Marlex mesh in one and sequestration of the sternum and clavicula in two patients. All these patients underwent surgical re-intervention. After removal of the Marlex mesh, complete wound granulation occurred within I month. Two patients with chronical sequestration needed three more operations to control osteoradionecrosis. One of them ultimately required a myocutaneous flap of the pectoralis major to reconstruct the thoracic defect 1.5 year after omental transposition. Two of the three patients who needed between one and three operations to control thoracic wound infection had osteoradionecrosis as indication for POSSG. Two patients had minor thoracic wound infection and were conservatively treated. There was no relation between the size of defect or the use of implants for chest wall reconstruction and the development of thoracic wound problems.

Nine patients (26/11) developed respiratory problems. Pneumothorax in four and pleural effusion leading to dyspnoea in three patients were conservatively treated. Reintubation followed by tracheostomy was necessary in one patient, who developed respiratory insufficiency after operation. One patient died 11 days after an operation for pulmonary embolism. All these respiratory complications occurred in nine patients with full thickness resection of the chest wall $(9 / 16=56 \%: P<0.001)$.

Of the nine patients who received additional therapy alter POSSG, one patient with irradical chest wall resection of a solt tissue sarcoma had complete POSSG failure 2 months after adjuvant radiation therapy (total doses $66 \mathrm{~Gy}$ ). Five months later a second stage procedure was performed with a myocutaneous flap of the pectoralis. In the other eight patients no problems relating to the POSSG were lound.

\section{Local ammotr and simptom control}

Twenty-four patients were treated with curative intention (Table 3). Of the 15 patients who were treated for breast cancer, 12 developed local culaneous metastases around the omentoplasty 14 days to 3.1 years (mean: 0.9 years $(S D=1.0$. $\mathrm{SEM}=0.3$ ) median: 0.5 years) alter POSSG. Duration of local tumour-free interval after POSSG is shown in Fig. 2. The 3-year overall survival after POSSG in this group of patients is $36 \%(\mathrm{SD}=14 \%)$ (Fig. 3). One patient with osteoradionecrosis as indication for chest wall resection developed local recurrence 2.7 years and died 5.0 years after POSSG. The other four patients are still alive 0.3 to 4.6 years after POSSG without evidence of disease.

Ten patients underwent palliative resection: seven patients with mastitis carcinomatosis and three with distant metastases. They all died within 0.2 to 1.9 years (mean: 0.9 years ( $\mathrm{SD}=0.6 . \mathrm{SEM}=0.2$ ) median: 0.5 years) of the operation (Fig. 3). Only one patient was free of local recurrence at the moment of his death 0.5 years after operation. The other nine patients developed local cutaneous metastases 0.1 to 0.4 years (mean: 0.2 years $(S D=0.1$ ) median: 0.1 years) after chest wall resection (Fig. 2). In three patients palliative resection was not successful. Within 6 weeks they had the same pre-operative problems of pain or bleeding due to local recurrence. In five patients the palliative effect of chest wall resection caused relief of local pain. bleeding or foetor until they died 6.4 to 22.3 months after POSSG (mean: 14.6 months, $S D=6.2$. $S E M=2.8$ ). Two patients had local symptom control until 3 to II weeks before death (survival 5.9 and 16.5 months after POSSG. respectively).

\section{Discussion}

Several authors recommend omentoplasty for reconstruction of partial-thickness chest wall defects after resection of radiation-injured tissue and for salvage of failed muscle flaps. In case of full thickness chest wall defects they advise the use 
Table 3. Curative resection in relation to local recurrence and mortality

\begin{tabular}{|c|c|c|c|c|c|}
\hline \multirow[t]{2}{*}{ Curative operation indication } & \multicolumn{2}{|c|}{ Local recurrence } & \multicolumn{2}{|r|}{ Death } & \multirow[t]{2}{*}{$N$} \\
\hline & $n$ & Median (years) & $n$ & Median (years) & \\
\hline Breast cancer & 12 & 0.5 & 10 & 2.4 & 15 \\
\hline Osteoradionecrosis & 1 & $\begin{array}{l}\text { not reached } \\
(>2.7 \text { years })\end{array}$ & 1 & 5.0 & 5 \\
\hline Soft tissue sarcoma & 2 & 0.3 & 2 & 0.8 & 4 \\
\hline Tolal & 15 & 0.7 & 13 & 3.0 & 24 \\
\hline
\end{tabular}

median $=$ median years after chest wall resection.

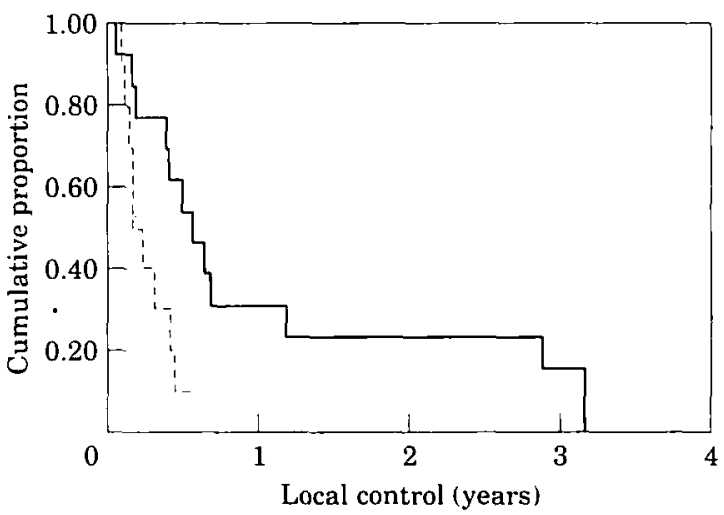

Fig. 2. Local tumour-free interval after curative (- - ) and palliative (---) chest wall resection. POSSG indication is recurrent breast cancer.

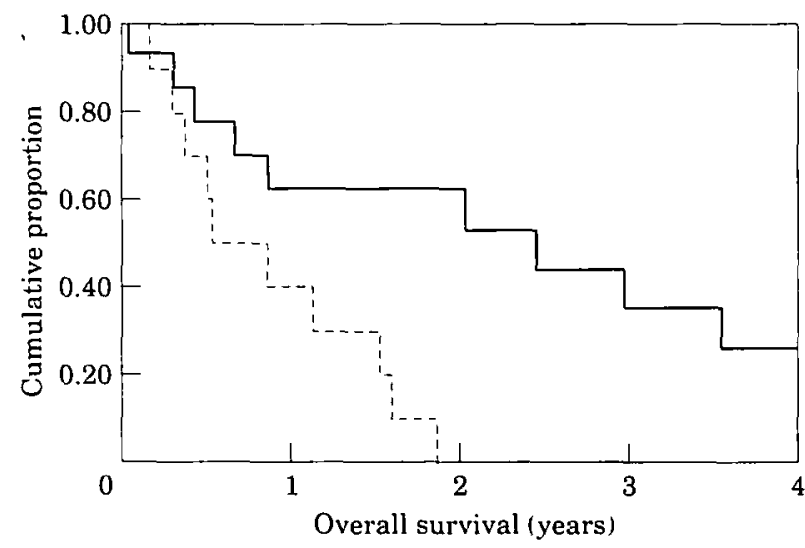

Fig. 3. Survival after curative (-) and palliative (--.) chest wall resection. POSSG indication is recurrent breast cancer.

of musculocutaneous flaps for chest wall reconstruction. 2.711 The main reasons for not using the omentum for reconstruction are the lack of rigidity ${ }^{7.10 .12}$ and the need for laparotomy." In our study 16 patients had skeletal resection of ribs and/or the sternum. In 13 patients the rigid defect was reconstructed by the use of implants. Two patients were given Marlex mesh for reconstruction, which had to be removed because of subsequent wound infection. Since the use of absorbable implants no persistent infectious complications of the thorax wound were seen. No ventilatory motion of the chest wall was observed in any of the patients. This confirms our opinion that when absorbable grafts are used in combination with POSSG excellent rigidity of the chest wall after lull thickness resection can be achieved. ${ }^{3}$ In our study instability of the remaining chest wall was never the case. However, long-term cosmetic result of homologous dura mater is disappointing without increased pulmonary complaints. In case of skeletal resection more pulmonary complications were seen. All patients except one, who needed long-term intubation for respiratory insufficiency, were conservatively treated. This underlines the importance of preoperative pulmonary lung function screening ${ }^{13}$ and other routine procedures usually needed in lung surgery.

An almost inevitable post-operative complication in exteriorized omentoplasties is incisional hernia. This fascial delect caused complaints in four patients: two were eflicaciously treated conservatively, two patients needed reoperation. To reduce the risk of incisional hernia, the vascular pedicle can be lead through a separate fascional incision. ${ }^{\text {it }}$

The repair of radiation injuries of the chest wall carries a high incidence of wound-healing complications. ${ }^{15}$ Based on experiences of flap reconstruction in radionecrosis some authors recommend omentoplasty for reconstruction. ${ }^{7 . s}$ The specific anti-inflammatory and angiogenitic agents produced in the omentum make it an excellent graft to reconstruct radiation-injured tissue. Wening et al. " operated on eight patients successfully for radionecrosis of the chest wall by omental transposition. Nevertheless, in our study two of five patients treated for radionecrosis needed between one and three more surgical interventions to control the severely infected wound. One of them suffered from a total flap failure, which was reconstructed by a pectoralis flap. In one recent study the latissimus dorsi musculocutaneous flap is considered to be the first-line flap for radiodystrophy treatment. compared with POSSG, fasciocutaneous flap and TRAM flap. " In general, complication rates for partial necrosis and prolonged thorax wound healing respectively after POSSG $\left(23-35^{(1 / 1)^{17} / 15-20 \%} / 0^{4}\right)$ and myocutaneous flaps $\left(24-36 \% 0^{15.19} / 7-16 \% \%^{7.14}\right)$ are roughly similar. In our study the most common complication after POSSG is partial necrosis (Table 2), which can be reduced by careful preparation. estimation of vascularization and a sufficiently wide subcutaneous tunnel. Little is written about the use of free vascular flaps for chest wall reconstruction. In one recent study, in which this technique was used to provide soft tissue cover after chest wall resection in seven patients, it was considered that this surgical procedure should be limited to cases with technical difficulty in using rotation or transposition flaps and to cases with minimal risk of 
infection. 21 The latter does not apply to our study, in which osteoradionecrosis or ulceration of the chest wall was often an indication for reconstruction.

Wide local excision provides better local control than other treatment modalities in patients with local recurrent breast cancer." Aggressive surgical treatment of local recurrent breast cancer without distant metastases can achieve a 3-year disease-free interval of $30-50 \%$. ${ }^{417.21}$

Patients undergoing palliative surgery show a poor prognosis for local tumour control (Fig. 2). Even for these patients this extended procedure can be defended in terms of control of ulceration, bleeding and foetor. which is also confirmed by other studies. ${ }^{16.22} 20$ In our study $70 \%$ of the patients treated with palliative intention are successfully locally treated until they dic (mean: 11 months after POSSG).

\section{Conclusion}

The defect after (full) thickness resection for local recurrence or primary malignancy of the chest wall can successfully be reconstructed with pedicled omentoplasty and split skin graft in combination with absorbable implants. Reconstruction following resection of radiation-induced necrotic tissue is prone to prolonged thoracic wound healing. In spite of successful results of the use of POSSG to reconstruct these defects in literature. our results are less convincing. Nevertheless, chest wall resection as a palliative procedure to control ulceration, bleeding and foetor can attain marked improvement in quality of survival. Unfortunately, in a small group of patients treated with palliative intention. large chest wall resection was not effective in controlling local symptoms due to recurrence of breast cancer. We could not differentiate these patients from the others in whom palliative resection caused local symptom control. This might be achieved by a multi-centre study. The choice of method of reconstruction differs in experience of surgeon and quality of donor site. In our opinion POSSG is a safe and time-saving procedure. whatever the indication of chest wall resection or wherever the acceptor site on the chest wall may be.

\section{References}

I. Kiricuta 1. I'Emploi du grand epiploon dans la chirurgie du sein cancereux. Press Medical 1963: 71:51-7.

2. Abbes M. Mateu J, Giordano P. Bourgeon $Y$. Chest wall reconstruction after full thickness resection: an experience with 22 patients. Eur J Surg Oncol 199I: 17: 342-9.

3. vin Geel AN. Wiggers Th. Eggermont AAM. Reconstruction of chest wall defects with homologous dura mater grafts. Br J Surg 1989: 76: 870 .

4. Zoetmulder FA, van Dongen JA. Chest wall resection in the treatment of local recurrence of breast cancer. Eur J Sirg Oncol 1988: 14: 127-32.

5. Kaplan EL. Meier P. Nonparametric estimation from incomplete observations. J Am Stul Assoc 1958; 53: 457-81.

6. van Geel AN, Wiggers Th. The pedicled omentoplasty: technical aspects in reconstructive and protective surgery. Neth J Sirrg 1991: 43: 150-3.

7. Arnold PG. Pailero PC. Chest wall reconstruction: experience with 100 consecutive patients. Ann Surg 1984; 199: 725-32.

8. Dingman RO. Argenta LC. Reconstruction of the chest wall. Ann Thorac Surg 1981: 32: 202-8.

9. Pairolero PC. Arnold PG. Chest wall reconstruction. Alm Thorac Surg 1981: 32: 325-6.

10. Sando W, Jurkiewicz MJ. An approach to repair of radiation necrosis of chest wall and mammary gland. World J Surg 1986; 10: $206-19$.

11. Wening JV. Thoma G. Emmerman A. Zornig C. Repair of infected defects of the chest wall by transposition of greater omentum. Br J Clin Pract 1990: 44: 311-3.

12. Larson DL. McMurtrey MJ. Musculocutaneous flap reconstruction of chest wall defects: an experience with 50 patients. Plast Reconstr Surg 1984: 73: 734-40.

13. Pairolero PC. Chest wall tumors: experience with 100 consecutive patients. J Thorac Cardiovasc Surg 1985; 90: 367-72.

14. Pitman MR, Lamont PM. Meiron TJ. Omental viability and incisional herniation after omental transposition for repair of the chest wall defects. Br J Surg 1988: 75: 72.

15. Kiricuta I. Use of Omentum in Plastic Surgert: Elmsford. NY: Pergamon Press, 1980: 14-30, 41-82. 97-124. 158-60, 270-8.

16. Rouanet P. Fabre JM. Tica V. Anal V. Jozwick M. Pujol H. Chest wall reconstruction for radionecrosis after breast carcinoma therapy. Alm Plast Surg 1995; 34: 465-70.

17. Williams RJ, Fryalt 1J. Abboll WC, White H. Omental transposition in the treatment of locally advanced and recurrent breast cancer. Br J Surg 1989: 76: 559-63.

18. Boyd AD. Shaw WW. McCartly JG. Bakker DC. Trehan NK, Acinapura AJ, Spencer FC. Immediate reconstruction of fullthickness chest wall defects. Anm Thorac Sirrg 1981: 32: 337-45.

19. Kroll SS, Schusterman MA. Larson DL. Fender A. Long-term survival alter chest-wall reconstruction with musculocutaneous flaps. Plast Reconsstr Surg 1990: 86: 697-701.

20. Al-Kaltan KM, Breach NM. Kaplan DK. Goldstraw P. Solttissue reconstruction in thoracic surgery. Afm Thorac Surg 1995; 60: $1372-5$.

21. Dahlstrom KK. Andersson AP. Andersen M. Krag C. Wide local excision of recurrent breast cancer in the thoracic wall. Cancer 1993: 72: 774-7.

22. Burnard RJ, Martini N, Beattie EJ. The value of resection in tumors involving the chest wall. I Thorac Cardiovasc Surg 1974: 68: $530-5$.

23. McCormack P. Bains MS. Beattie EJ, Martini N. New trends in skeletal reconstruction after resection of chest wall tumors. Amm Thorac Surg 1981: 31: 45-52.

24. Muscolino G. Valente M. Lequaglie C, Ravasi G. Correlation between first disease-free interval from mastectomy to second disease-free interval from chest wall resection. Eur J Sirg Oncol 1992: 18: 49-52.

25. Noguchi S. Miyauchi K, Nishizawa Y, Imaoka S, Koyama $H$, Iwanaga $T$. Results of surgical treatment for sternal metastasis of breast cancer. Cancer 1988: 62: 1397-401.

26. Ryan MB, McMurtrey MJ, Roth JA. Current management of chest-wall tumors. Sturg Clin North .4m 1989; 69: 1061-80.

Accepted for publicution $3 \mathrm{Jull} / 996$ 